\title{
Le Québec en Amérique du Nord: Genèse d'une société nouvelle ${ }^{*}$
}

\author{
Fernand HARVEY \\ Professeur et titulaire \\ Chaire Fernand-Dumont sur la culture \\ Institut national de la recherche scientifique \\ Québec
}

Depuis la rencontre entre Champlain, Gravé du Pont et le chef amérindien Anadabijou à Tadoussac, en 1604, quatre siècles se sont écoulés au cours desquels le fait français a pris racine dans le Nouveau Monde, malgré les vicissitudes de l'histoire. Considérée à l'échelle de la France et de l'Europe, cette présence française sur le vaste continent de l'Amérique du Nord a quelque chose de démesuré que les dirigeants politiques des $16^{\mathrm{e}}$ et $17^{\mathrm{e}}$ siècles ne pourraient sans doute pas mesurer géopolitiquement dans toute son ampleur, ni apercevoir dans le futur lointain. Même de nos jours, envisager une interprétation d'ensemble du fait français en Amérique, à partir du Québec, du Canada français, de l'Acadie, de la Nouvelle-Angleterre, du Middle West américain, de la Louisiane, de la Californie, d'Haïti et des Antilles françaises relève pour l'instant d'un souhait plus que d'une réalité, compte tenu de l'état de nos réflexions et d'un certain cloisonnement de nos intérêts. Mais de tels rapprochements s'imposent plus naturellement dans le nouveau contexte de la mondialisation et des débats sur la diversité culturelle. Vous me permettrez de jeter un regard sur un aspect de cette vaste mosaïque, à savoir l'itinéraire historique et contemporain du Québec et du Canada français en Amérique.

Fasciné par une Amérique du Nord à peine explorée, Champlain se veut visionnaire d'une importante colonie de peuplement français, même si l'intérêt des marchands qui le soutiennent s'oriente en fonction du commerce des fourrures, plutôt que d'un enracinement de type agricole. Par ailleurs, le père de la Nouvelle-France a vite compris, sans doute mieux que l'explorateur français Jacques Cartier, en 1534, qu'un établissement stable dans la vallée du SaintLaurent et une pénétration à l'intérieur du continent ne saurait faire l'économie

- Communication présentée à la Catedra Margaret Atwood-Gabrielle Roy de estudios canadienses, Facultad Filosofia y Letras de la Universidad Nacional Autonoma de Mexico, Mexico, lundi, 11 avril 2005. 
d'une alliance avec certains peuples autochtones. À la fin de sa vie, l'œuvre colonisatrice de Champlain demeurait bien modeste par rapport aux rêves de développement qu'il avait caressé. Mais il avait fondé les bases d'un rapport à la territorialité qui allait marquer, non seulement la Nouvelle-France, mais le peuple canadien et québécois qui en émergeraient par la suite. Champlain est une figure double: d'une part l'explorateur, le cartographe et le diplomate qui établit des rapports avec l'Autre, en l'occurrence l'Amérindien, et d'autre part le colonisateur et le gouverneur qui s'emploie à établir et à développer un noyau de peuplement d'origine française et européenne (Litalien et Vaugeois, 2004).

Ce dualisme des origines va se perpétuer au cours des siècles qui suivront en s'adaptant aux circonstances et aux nouveaux rapports de force créés par l'histoire.

Sous le régime français et jusqu'à la conquête anglaise de 1760 , on verra ainsi se développer deux Nouvelle-France: celle des seigneuries de la vallée du Saint-Laurent -une longue bande de terres défrichées de 400 kilomètres entre Kamouraska, à l'est de Québec et Montréal, à l'ouest- et celle des explorations et de la traite des fourrures à l'intérieur du continent vers la Baie d'Hudson, les Grands Lacs et la vallée du Mississipi jusqu'en Louisiane; sans compter la mouvance vers l'Ouest du continent. En somme, une dialectique entre l'enracinement et la mobilité (Louder et Waddell, 1983). Du côté de l'Atlantique, une colonisation sœur, celle de l'Acadie, se développera plus modestement et plus difficilement à cause des rivalités anglo-françaises et se verra imposer bien malgré elle un autre dualisme beaucoup plus menaçant pour son identité naissante, celui de l'exil ou du retour, à la suite du traumatisme de la Déportation de 1755 (Harvey et Beaulieu, 2000).

Sous le régime anglais, le rapport avec l'Autre change radicalement pour les Canadiens de la vallée du Saint-Laurent. L'Amérindien est marginalisé et fait place à l'Anglais, nouveau colonisateur du pays qui domine une économie axée sur le commerce des fourrures, puis à partir du début du $19^{\mathrm{e}}$ siècle, sur l'industrie du bois. Ce nouveau rapport engendre une profonde mutation politique et institutionnelle chez les Canadiens natifs du pays et qu'on appellera par la suite French Canadians. L'introduction par le gouvernement impérial anglais des institutions parlementaires britanniques dans le Bas-Canada en 1791 (l'actuelle province de Québec), malgré l'absence de la responsabilité ministérielle avant 1849 , permettra à une nouvelle élite politique canadiennefrançaise de s'affirmer et de contrôler la Chambre d'Assemblée, compte tenu du fait que les Canadiens français constituaient $80 \%$ de la population de la province jusqu'en 1840. Ni l'écrasement des Rébellions de 1837-1838 qui venait anéantir les projets d'indépendance politique du Bas-Canada caressés par Louis-Joseph Papineau et son Parti Patriote, ni l'Acte d'Union du Haut et du 
Bas-Canada de 1840 qui consacrait la mise en minorité des Canadiens français dans le nouveau régime du Canada-Uni, puis celui de la Confédération de 1867, ne viendront entamer la capacité de survie politique des Canadiens français en tant que nation et société distincte à l'intérieur du Canada et en Amérique du Nord. Les négociations ayant abouti à la Constitution fédérale de 1867 ont en effet permis aux Canadiens français résidant au Québec de contrôler un état provincial, même si cet espace politique est longtemps demeuré affaibli par le jeu des partis et l'absence de leviers économiques pouvant servir les intérêts de la majorité francophone (Lacoursière, Provencher et Vaugeois, 2000).

Cette capacité politique, même limitée, correspondant à une certaine idée de la nation, à laquelle s'ajoutera une capacité économique et culturelle nouvelle, à partir de la Révolution tranquille, explique pourquoi les Canadiens français de jadis, et les Québécois d'aujourd'hui ne peuvent être considérés comme une minorité comme les autres au Canada et en Amérique du Nord. Cette réalité politique fondamentale est difficilement compréhensible pour un observateur extérieur ignorant l'histoire du Canada.

Au cours des $19^{\mathrm{e}}$ et $20^{\mathrm{e}}$ siècles, la petite société ancrée depuis ses origines dans la vallée du Saint-Laurent a progressivement développé ses institutions, son économie, ses régions périphériques de colonisation, ses villes, ses références culturelles, non sans de longues périodes d'hésitation ou de stagnation, longtemps sous la tutelle du clergé catholique et du capital anglo-américain (Dickinson et Young, 1995).

Parallèlement à cette société canadienne-française de l'enracinement territorial s'en développait une autre: celle de la mouvance, des migrations et des diasporas francophones vers l'Ontario, l'Ouest canadien, la Nouvelle-Angleterre et le Middle West américain, au cours du $19^{\mathrm{e}}$ siècle et jusqu'à la veille de la seconde guerre mondiale.

Dans ce contexte de mouvance, on peut distinguer deux types de migrations. D'abord celle des individus: les voyageurs employés par la Compagnie de la Baie d'Hudson et la Compagnie du Nord-Ouest parcourant l'Ouest canadien et donnant naissance à la nation Métis de Louis Riel. À titre individuel, on retrouve également les aventuriers de toutes sortes participant aux premières expéditions dans le Grand Nord canadien ou dans l'Ouest américain. Certains y ont laissé leur marque jusqu'en Californie (Lamontagne, 2002). L'autre type de migration se caractérise plutôt par son côté familial et communautaire et s'inscrit dans un double mouvement: l'industrie manufacturière et de colonisation agricole. Les emplois à bas salaire, les Canadiens-français de la seconde moitié du $19^{\mathrm{e}}$ siècle sont allés les chercher dans les fabriques de textiles de la Nouvelle-Angleterre, mais ainsi dans les villes industrielles du Middle West américain et dans les villes minières du Nord de l'Ontario. Pendant ce temps 
et concurremment, d'autres fondaient des communautés rurales dans l'est et le nord de l'Ontario ou dans l'Ouest canadien, à une époque ou l'agriculture demeurait encore l'activité dominante au Canada; elle le demeurera, du reste, jusqu'aux années 1920-1930 (Jaenen, 1993).

Cette diaspora canadienne-française issue de la vieille province de Québec et établie ailleurs au Canada et aux États-Unis était encadrée par des institutions patriotiques et religieuses basées sur la langue française et la foi catholique. Entre le Québec et ces communautés canadiennes-françaises et acadiennes s'était développé un vaste réseau de solidarité animé par l'idéologie de la survivance française et de la mission civilisatrice du Canada français au sein de l'Amérique anglo-saxonne et protestante. Le clergé catholique, par ses écoles, ses collèges et ses paroisses, a joué un rôle essentiel pour maintenir la cohésion de l'ensemble, du moins jusqu'à la grande crise économique des années 1930 (Roby, 1887, 1990).

L'éclatement du Canada français traditionnel, à la faveur de la Révolution tranquille au Québec, dont on a fait grand cas, était amorcé bien avant. On en perçoit un indice bien réel avec l'assimilation rapide de la plus importante diaspora canadienne-française, celle des Franco-Américains, dès les années 1930. Écartelés entre leur identité canadienne-française d'origine et leur nouvelle identité américaine et contraints à abandonner leurs institutions scolaires francophones, ces Petits Canadas, ou ce "Québec d'En-bas" comme on l'a appelé plus récemment, a rapidement perdu ses liens familiaux et ses repères culturels avec son ancienne mère patrie. Devenir Américains constituait un objectif naturel pour les nouvelles générations. Au Canada anglais, pendant ce temps, les communautés canadiennes-françaises de l'Ontario des provinces de l'Ouest ont maintenu tant bien que mal leurs positions, malgré les ravages de l'assimilation et les contraintes historiques des lois scolaires provinciales à l'égard des écoles françaises et catholiques (Thériault, 1999; Louder, 1991; Roby, 1990).

Que s'est il passé entre le Québec français et sa diaspora historique à partir des années 1960? La représentation du Canada français continental et pancanadien des décennies antérieures s'est effondrée. La tenue à Montréal, en 1967, d'un grand rassemblement national, les États généraux du Canada français, a marqué une rupture symbolique de ce réseau culturel et institutionnel canadienfrançais qui s'étendait à toutes les provinces canadiennes et jadis également à la Nouvelle-Angleterre. Allait s'ensuivre une longue période d'éloignement et de refroidissement des relations entre le Québec et les communautés francophones minoritaires du Canada (Harvey, 1995; Martel, 1997).

Pendant trois décennies et jusqu'au seuil des années 1990, le Québec, tant au niveau de l'État que de la société civile, s'est employé à moderniser ses institutions politiques et administratives, à s'assurer du contrôle progressif de 
son économie et à redéfinir les paramètres de sa culture et de ses relations avec le Canada anglais (Linteau et al., vol. 2, 1989). Dans un premier temps cette nouvelle affirmation de soi des années 1960, à caractère géopolitique (le territoire québécois), plutôt qu'ethnoculturel, comme dans l'ancien Canada français, laissait dans l'ombre les rapports avec les Amérindiens, avec les immigrants installés au Québec au cours du $20^{\mathrm{e}}$ siècle, et aussi avec les francophones hors Québec, pour ne retenir que les rapports conflictuels avec le Canada anglais et le gouvernement fédéral. Mais la question de l'immigration est par la suite devenue centrale au cours des années 1970. Comment faire en sorte que les immigrants et les communautés ethnoculturelles vivant à l'intérieur du Québec s'intègrent à la société francophone majoritaire plutôt de se s`assimiler dans une proportion de $85 \%$ à la culture anglophone via la fréquentation des écoles anglaises? La Charte de la langue française adoptée par l'Assemblée nationale en 1977 a voulu renverser la vapeur en obligeant les enfants francophones et immigrants à s'inscrire à l'école française. La loi 101, comme on l'appelle communément, a eu des effets positifs que tous reconnaissent au Québec. En plus d'assurer la paix linguistique et de respecter les droits historiques de la minorité anglaise, elle a jeté les bases d'un nouveau pluralisme culturel axé sur l'usage d'une langue commune: le français (Pâquet, 2005).

Par ailleurs, les nouveaux rapports avec les peuples autochtones ont été plus lents à s'esquisser. Les grandes alliances du régime français amorcées par Champlain au début du $17^{\mathrm{e}}$ siècle avaient été élargies lors de la Grande Paix de Montréal, en 1701, entre les Français et tous le peuples autochtones de l'intérieur du continent (Havard, 1992). Cette alliance s'était confirmée durant la guerre de Sept Ans précédant la conquête anglaise de 1759, puisque plusieurs groupes d'Amérindiens avaient combattu aux côtés des Français et des Canadiens contre les Anglais et les Américains. Après 1760, le régime anglais a eu pour conséquence d'isoler les Canadiens français et de marginaliser les peuples autochtones, puisque ceux-ci n'étaient plus des alliés stratégiques pour le contrôle de l'intérieur du continent nord-américain. Cette longue mise entre parenthèses historique allait durer quelque 240 ans. Pendant ce temps, les Amérindiens étaient pris en charge par le gouvernement britannique jusqu'en 1867 , puis par le nouveau gouvernement fédéral canadien par la suite et parqués dans des réserves.

La Paix des Braves signée en 2002 entre le Québec et la nation Cris marque symboliquement et concrètement le retour sur la scène historique des rapports entre la société québécoise et les peuples autochtones. Cette entente accordait aux Cris un rôle actif dans le développement des richesses naturelles et hydroélectriques du vaste territoire de la Baie James. Et bonifiait pour eux la Convention de la Baie James signée en 1975. Beaucoup de chemin reste à 
faire entre le Québec et les autres communautés autochtones, mais le parcours est maintenant mieux balisé.

À l'intérieur du Québec, de nouveaux rapports marqués par un rapprochement culturel se sont également développés entre le Québec français et sa communauté anglophone, plus particulièrement à Montréal, devenue le creuset d'une nouvelle culture plurielle.

Tous ces processus de réaménagement des rapports d'altérité et de pluralité à l'intérieur du Québec ont laissé plus ou moins dans l'ombre les rapports avec les communautés francophones minoritaires hors Québec, cet archipel de l'ancienne mouvance migratoire issue de la vallée du Saint-Laurent et de l'Acadie. Il faut dire que ces rapports n'ont jamais été rompus, mais qu'ils ont connu une longue période de turbulence à l'intérieur du Canada compte tenu des positions politiques opposées qui ont séparé le Québec nationaliste et une bonne partie des leaders des communautés francophones et acadiennes, lors des débats constitutionnels canadiens et des deux référendums sur l'avenir du Québec, entre 1976 et 1995. L'adoption en 1993 par le gouvernement du Québec d'une première politique officielle d'aide et de collaboration avec les communautés francophones et acadiennes marque un tournant par rapport à la situation des trois décennies précédentes, même si ce rapprochement souhaité au niveau de la société civile demeure bien timide et qu'il se fait discret dans les débats intellectuels.

Il importe cependant d'observer aussi ce qui se passe sur le terrain et d'esquisser la géographie culturelle de ces communautés francophones et de leurs rapports avec le Québec. Une telle exploration exigerait cependant de longs développements. Qu'il suffise ici de rappeler que les communautés francophones et acadiennes du Canada ont connu une importante mutation sociale et culturelle parallèle à celle du Québec. De majoritairement rurales qu'elles étaient au départ, elles sont devenues urbaines avec les facteurs aggravants d'assimilation que cela implique; leur identité canadienne-française d'origine s'est transformée en identités francophones provinciales: on se définit maintenant comme Franco-Ontariens, Franco-Manitobains, Franco-Albertains, etc. Seuls les Acadiens des provinces maritimes ont conservé une identité nationale qui leur est propre. Supportées financièrement par le gouvernement fédéral, ces communautés ont, par ailleurs, consolidé leurs assises associatives et se sont vues accorder des droits à la gestion de leurs écoles par la Cour Suprême du Canada. Dans le domaine littéraire et artistique, un nouvel espace de création et d'expression développé à partir des années 1970 au sein des communautés francophones a souvent eu des échos jusqu'au Québec, particulièrement dans le cas des artistes et écrivains acadiens. Dans ce nouveau contexte de développement des industries culturelles francophones, Montréal s'est affirmé comme la métropole culturelle non seulement 
du Québec, mais aussi des communautés francophones minoritaires du Canada (Cardinal, 1994; Behiels, 2005).

La continentalisation et la mondialisation des échanges pose maintenant de nouveaux défis culturels sans rapport avec une vision nostalgique de l'ancien Canada français. La capacité pour les francophones d'Amérique de participer à la nouvelle société du savoir et de s'appuyer sur de multiples réseaux engendrés ou soutenus par les nouvelles technologies d'information et de communication pourrait contribuer à résoudre l'ancien dilemme entre une mouvance individuelle ou collective à l'intérieur du continent, voire même au-delà. Mais tout réseau implique une tête de réseau. À cet égard, le Québec, par sa masse critique, s'impose comme l'incontournable tête de ce réseau, mais dans un esprit d'ouverture et de collaboration avec les autres communautés francophones du Canada et des États-Unis. Au-delà de cet horizon continental, on peut déjà apercevoir le développement de nouveaux réseaux de solidarité et d'échange impliquant les Antilles, le Mexique et toute l'Amérique latine.

\section{Bibliographie}

BEHIELS, Michael. 2005. La Francophonie canadienne. Renouveau constitutionnel et gouvernance scolaire. Traduit François GAUTHIER. Ottawa: Presses de l'Université d'Ottawa.

CARDINAL, Linda. 1994. "Ruptures et fragmentations de l'identité francophone en milieu minoritaire; un bilan critique”. Sociologie et Sociétés, 26, 1. 71-86.

COUTURIER, Jacques Paul. 1996. Un passé composé. Le Canada de 1850 à nos jours. Moncton: Éditions d'Acadie.

DELAGE, Denys. 1991. Le pays renversé. Amérindiens et Européens en Amérique $d u$ Nord Est, 1600-1664. Montréal: Éditions du Boréal.

DICKINSON, John A. et Brian YouNG. 1995. Brève histoire socio-économique $d u$ Québec. $2^{\mathfrak{e}}$ éd. Sillery: Éditions du Septentrion.

HAMELIN, Jean. 1976. Histoire du Québec. St-Hyacinthe et Toulouse: Edisem et Privat.

HARVEY, Fernand. 1995. "Le Québec et le Canada français: histoire d'une déchirure". Dans Simon Langlois, dir. Identités et cultures nationales. L'Amérique française en mutation. (Québec: Presses de l'Université Laval. 49-62.

et Gérard BEAULIEU, dir. 2000. Les relations entre le Québec et l'Acadie, de la tradition à la modernité. Québec: Éditions de l'IQRC / Presses de l'Université Laval.

HAVARD, Gilles. 1992. La Grande Paix de Montréal de 1701: les voies de la diplomatie franco-amérindienne. Québec: Recherches Amérindiennes. 
JAEnEN, Cornelius, dir. 1993. Les Franco-Ontariens. Ottawa: Presses de l'Université d'Ottawa.

LACOURSIÈRE, Jacques, Jean PROVENCHER et Denis VAUGEOIS. 2000. CanadaQuébec. Synthèse historique, 1534-2000. Sillery: Éditions du Septentrion. Ill.

LAMONTAGNE, Sophie-Laurence. 2002. Canadiens-français et Québécois en Californie. Québec: Institut National de la Recherche Scientifique, Centre Urbanisation, Culture et Société.

LINTEAU, Paul-André, René DUROCHER et Jean-Claude ROBERT. 1989. Histoire du Québec contemporain. Montréal: Éditions du Boréal. 2 vols.

LITALIEN, Raymonde et Denis VAUGEOIS, dir. 2004. Champlain: la naissance de l'Amérique française. Québec: Éditions Septentrion. Ill. Cartes.

LOUDER, Dean, dir. 1991. Le Québec et les francophones de la Nouvelle-Angleterre. Québec: Presses de l'Université Laval.

et Éric WADDELL, éd. 1983. Du continent perdu à l'archipel retrouvé: le Québec et l'Amérique française. Québec: Presses de l'Université Laval.

MARTEL, Marcel. 1997. Le deuil d'un pays imaginé. Rêves, luttes et déroute du Canada français. Ottawa: Presses de l'Université d'Ottawa.

PÂQUET, Martin. 2005. Tracer les marges de la cité. Étranger, immigrant et État au Québec, 1627-1981. Montréal: Éditions du Boréal.

RoBY, Yves. 1987. "Quebec in the United States: A Historiographical Survey". Main Historical Society Quaterly, 26, 3 (hiver). 126-159.

1990. Les Franco-Américains de la Nouvelle-Angleterre, 1776-1930. Sillery: Éditions du Septentrion.

THÉRIAULT, Joseph-Yvon, dir. 1999. Francophonies minoritaires au Canada. L'état des lieux. Moncton: Éditions d'Acadie. 